\title{
Glenohumeral Rotational Angles May Affect Outcomes of Cross- Body Stretch: A Randomised Controlled Crossover Pilot Trial
}

\author{
Koya Mine ${ }^{1,2^{*}}$ \\ ${ }^{1}$ Department of Physical Therapy, Tokyo University of Technology, Japan \\ ${ }^{2}$ International Centre for Allied Health Evidence, University of South Australia, Australia

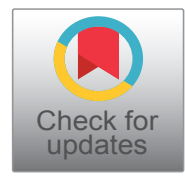 \\ *Corresponding author: Koya Mine, Department of Physical Therapy, School of Health Sciences, Tokyo University of Tech- \\ nology, 5-23-22 Nishi-kamata, Ota ward, 144-8535, Tokyo, Japan, Tel: +81-364242210
}

\begin{abstract}
Objectives: Various stretching techniques have been proposed to prevent or improve glenohumeral internal rotation deficit (GIRD) and posterior shoulder tightness (PST). Cross-body stretch is one of the most common methods to address GIRD and PST. The current evidence is unclear with regards to the influences of glenohumeral rotational angle during cross-body stretch. This study aimed to compare the effects of cross-body stretch in neutral rotation and 90-degree external rotation (ER).
\end{abstract}

Methods: This study was a crossover randomised controlled trial. 10 young healthy individuals (nine men and one woman, age $21.1 \pm 0.3$ years, body mass index $20.9 \pm 1.8$ ) with GIRD participated in this study. At two separate sessions, participants actively performed randomly assigned stretching techniques. range of motions (ROM) of dominant shoulder in internal rotation (IR) and horizontal adduction (HA) were assessed before and after stretching interventions. Stretching sensation and shoulder pain during stretches were also assessed using visual analogue scale after the interventions.

Results: Only cross-body stretch in 90-degree ER led to significant immediate improvements in IR ROM ( $p<0.05)$. There was no significant improvement in HA ROM after the two stretching interventions. There was no significant difference between the two intervention groups in terms of ROM changes. Cross-body stretch in neutral rotation provoked significantly more shoulder pain $(p<0.05)$.

Conclusions: Cross-body stretch in 90-degree ER might be more likely to be effective to improve IR ROM immediately among asymptomatic adults with GIRD with less chance of provoking shoulder pain. The findings also suggest that either form of cross-body stretch may not be effective to improve PST immediately. Further study with a larger sample size is required to achieve stronger evidence with better statistical precision and consider its clinical implication.

\begin{abstract}
Keywords
Cross-body stretch, Glenohumeral internal rotation deficit, Posterior shoulder tightness, Crossover randomised controlled trial
\end{abstract}

\section{Introduction}

Glenohumeral internal rotation deficit (GIRD) and posterior shoulder tightness (PST) have been reported in the contexts of various overhead sports, such as baseball, cricket, volleyball and tennis [1-4]. GIRD has been generally defined as decreased internal rotation (IR) range of motion (ROM) in the dominant arm compared to the non-dominant side [5]. PST can be evaluated as passive shoulder horizontal adduction (HA) ROM with the scapula stabilized in supine or side lying [6]. Since both PST and GIRD are thought to reflect stiffness of posterior structures in the shoulder, the term PST is occasionally used instead of GIRD in order to describe decreased shoulder IR ROM [7].

Although the aetiology and the mechanism of GIRD and PST are still elusive, some studies postulated that these physical impairments can be caused by chronic tensile loading through repetitive throwing activities, leading to microtrauma, scar formation and thickening of the posterior joint capsule [8]. In fact, one cadaveric study showed that simulated posterior capsule contracture can lead to GIRD [9]. It has been also suggested that repetitive strain in posterior muscles in the follow through phase in throwing motions can result in increased muscle stiffness [8]. One cross-sectional study

Citation: Mine K (2018) Glenohumeral Rotational Angles May Affect Outcomes of Cross-Body Stretch: A Randomised Controlled Crossover Pilot Trial. Int J Sports Exerc Med 4:103. doi.org/10.23937/24695718/1510103

Accepted: August 25, 2018; Published: August 27, 2018

Copyright: (c) 2018 Mine K. This is an open-access article distributed under the terms of the Creative Commons Attribution License, which permits unrestricted use, distribution, and reproduction in any medium, provided the original author and source are credited. 
found significant correlations between GIRD and stiffness in posterior deltoid, infraspinatus and teres minor muscles in patients with shoulder pain [10]. In addition to these soft tissue changes, GIRD can be also influenced by humeral retrotorsion as a bony adaptation among those who participated in overhead sports at younger ages [11].

A body of evidence regarding contributions of GIRD and PST to shoulder pain is compelling. The presence of GIRD and PST has been reported to be associated with subacromial impingement symptoms [12]. One cadaveric study found that PST can lead to a significant increase in peak subacromial contact pressure during shoulder motions [13]. Symptomatic internal impingement, the other form of impingement is most commonly found in baseball, where approximately 184-degree external rotation (ER) can occur in the late cocking phase of pitching [14]. A prospective cohort study reported that high school baseball and softball players with GIRD appeared to have higher risks for shoulder injuries compared to those without GIRD [15]. Another prospective cohort study reported that the resolution of shoulder symptoms was associated with correction of PST in athletes with internal impingement symptoms [16].

Various stretching interventions have been proposed to address GIRD and PST. One of the most common forms of stretching techniques is cross-body stretch. In cross-body stretch, patients horizontally adduct one shoulder with the contralateral hand in sitting or standing. It can be performed either actively or passively. This stretching method has been found to be effective to improve GIRD and PST in the short term $[17,18]$. To the best of the author's knowledge, however, no study specified or mentioned glenohumeral rotational angles during cross-body stretch. It can be argued that this element can be important because performing cross-body stretch with glenohumeral joint in IR position can put more tensile stress to posterior tissue as well as more compressive load to subacromial tissues theoretically, therefore it can affect the outcomes of the stretch. The lack of specification with regards to glenohumeral rotational angles during cross-body stretch might have confounded the findings in previous studies which investigated the effects of this stretching intervention $[19,20]$. The lack of clear evidence and uncertainty regarding the influences of glenohumeral rotational angles is concerning for all evidence-based clinicians who manage patients or athletes with GIRD and PST.

The purpose of this pilot study was to examine the influences of glenohumeral rotation angles on crossbody stretch among asymptomatic participants.

\section{Methods}

\section{Ethical approval and trial protocol}

This research was approved by an ethical committee at Tokyo University of Technology before the commencement of experiments (registration number: E17HS-014). Prior to data collection, all participants were provided with information regarding the risks and benefits of taking part in the study. Each participant volitionally signed an informed consent document. All participants were able to withdraw at any stage of the experiments. The protocol of this trial was registered in University Hospital Medical Information Clinical Trials Registry (registration number: UMIN000031974) in advance.

\section{Participants}

10 healthy young collegiate students (nine men and one woman, age $21.1 \pm 0.3$ years, body weight $60.7 \pm 6.9$ $\mathrm{kg}$, height $170.3 \pm 4.9 \mathrm{~cm}$, body mass index $20.9 \pm 1.8$ ) with GIRD were recruited for this study. Before initiating data collection, screening tests were taken to identify participants with GIRD. In this study, GIRD was defined as more than $15^{\circ}$ decrease in IR at 90-degree abduction in the dominant side compared to the non-dominant side. Subjects were excluded if they met any of the following exclusion criteria: (1) recent history of shoulder pain and (2) past history of surgery in the shoulder.

A sampling process is shown in Figure 1. At first, 17 healthy collegiate students volunteered to participate in this research. Following screening assessments, seven students were excluded due to the lack of GIRD. All 10 eligible participants were right-handed. The majority of the participants had experiences in overhead sports, including baseball $(n=4)$, tennis $(n=1)$ and swimming ( $=1)$. Other sports included football $(n=2)$, table tennis $(n=1)$ and karate $(n=1)$. All participants were instructed to refrain from any vigorous overhead exercises or shoulder stretching for 24 hours before sessions. Due to the nature of the stretching interventions, it was not possible to blind participants, who also served as intervention providers in this study.

\section{Experimental procedures}

This study was a crossover randomised controlled trial, in which each participant completed two experimental sessions in two separate days. The cross-body stretch was performed by participants actively in either glenohumeral neutral rotation or 90-degree ER (Figure 2). Elbow joint was maintained in 90-degree flexion to enable participants to monitor glenohumeral rotational angles easily. They performed stretching in a seated position without a back rest for support. They were instructed to hold the end position statically for 20 seconds and repeat it five times with a 10-second rest between stretches. Thus, each stretching intervention lasted for two minutes and 20 seconds in total. The two stretching methods are illustrated in Figure 2.

After the recruitment of 10 participants, each participant completed two experiments in a random order, which enabled an allocation concealment (Figure 1). Two testing sessions were separated by a minimum of 24 hours, in 


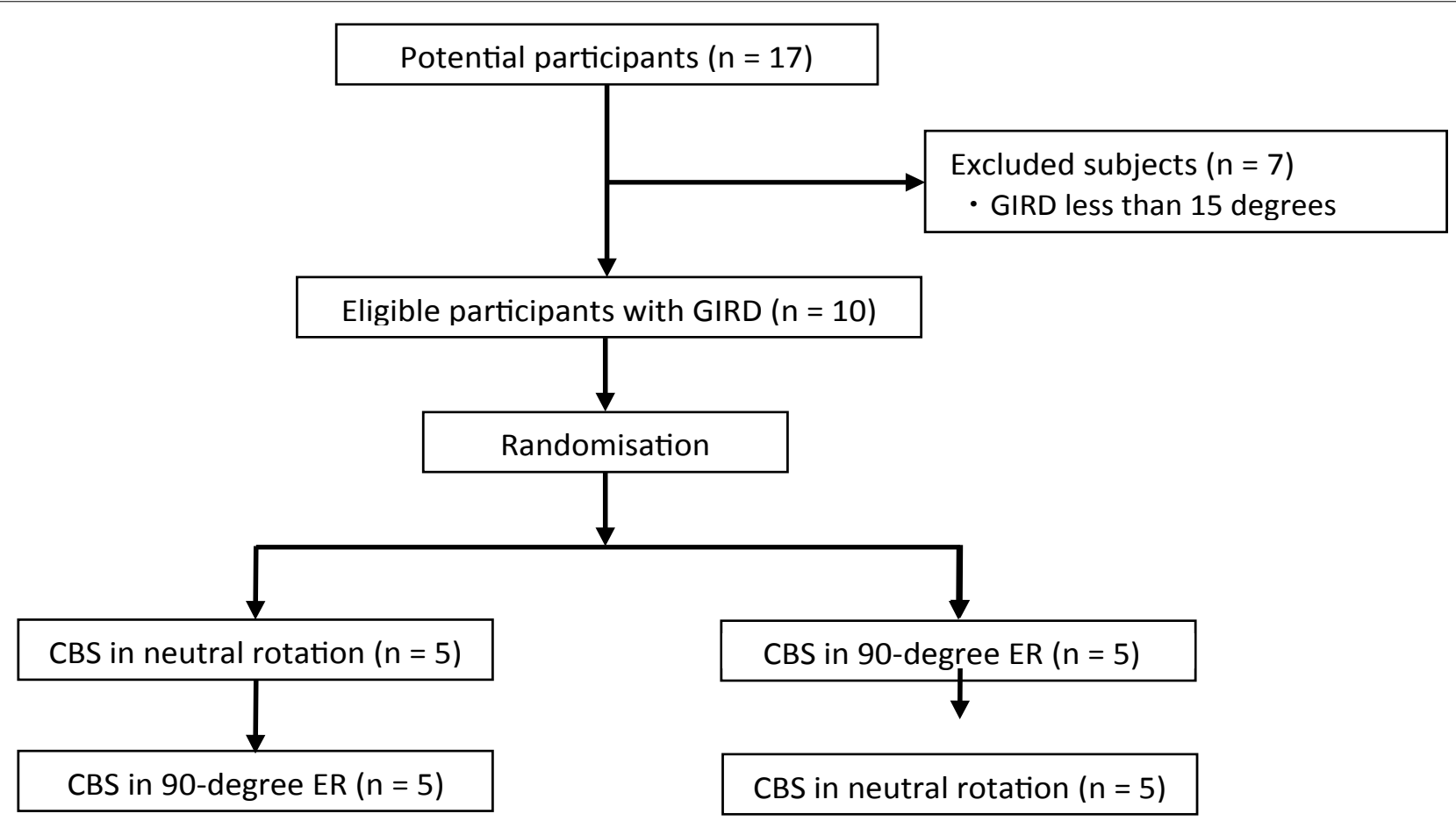

Figure 1: A flowchart of the study. CBS = Cross-body stretch.
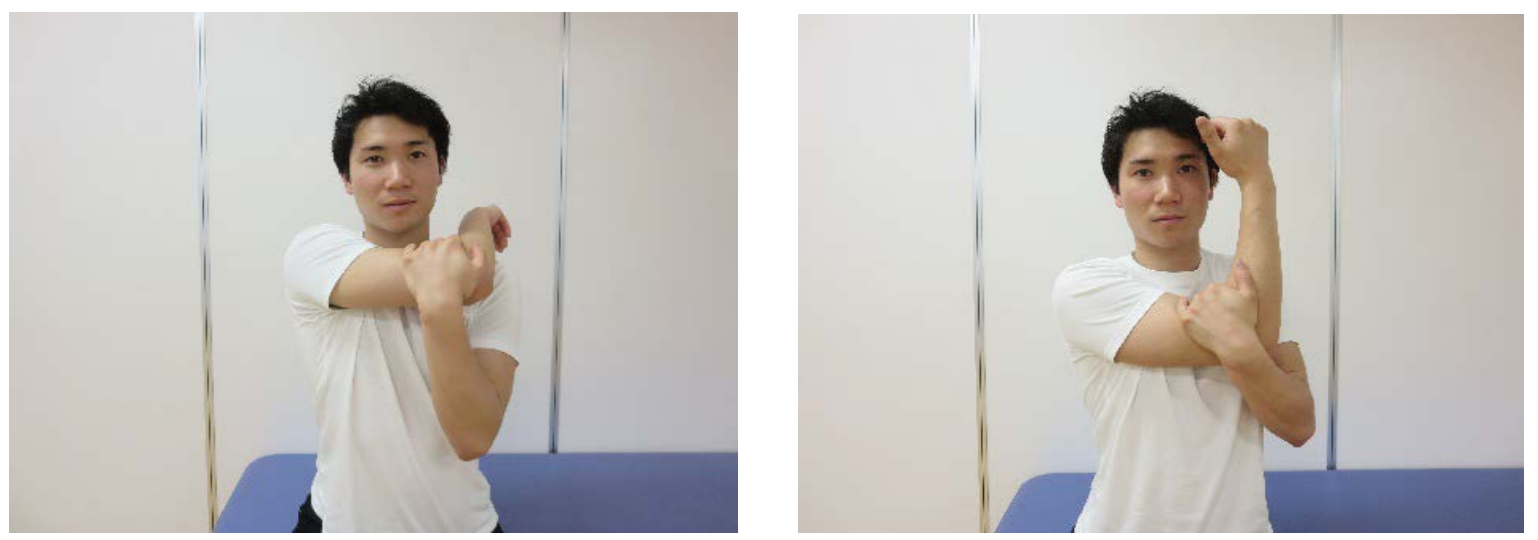

Figure 2: Methods of stretching techniques; cross-body stretch in neutral rotation (left) and cross-body stretch in 90-degree ER (right).
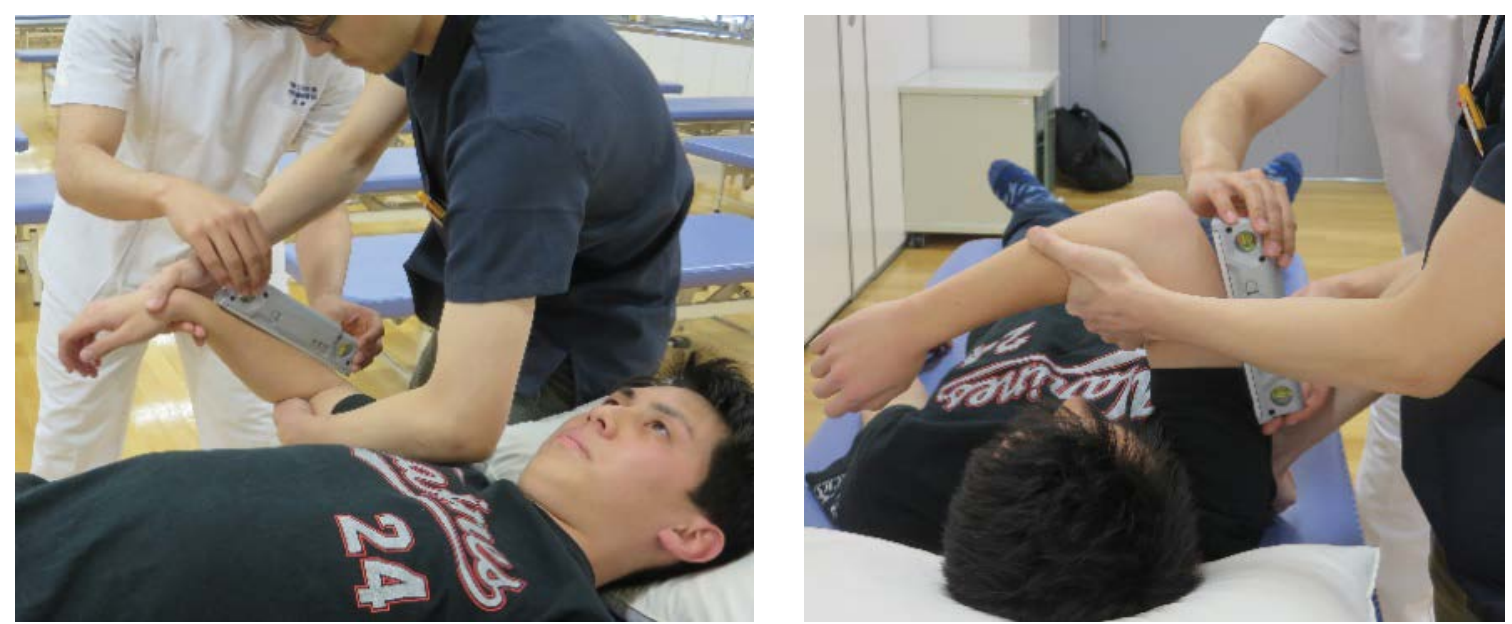

Figure 3: Measurement methods; IR (left) and HA (right).

order to minimise potential carry-over effects of the stretches. In both intervention sessions, the baseline data for IR and HA on the dominant side was collected in this order. ROM measurements were performed by the same two examiners (one qualified physiotherapist and one undergraduate physiotherapy student) using a 
digital inclinometer (Myzox, Japan) (see Figure 3). The primary assessor, who had completed master's degree in musculoskeletal and sports physiotherapy, passively moved participants' shoulder into end range with maximum resistance. The secondary assessor was responsible for placement of the inclinometer for all measurements taken. Maximum ROM was defined as when the primary assessor experienced maximum resistance to further glenohumeral motion while the shoulder girdle was manually stabilized (see Figure 3 ). The assessments were performed for each participant lying in supine on the same treatment table without a pillow. IR ROM was assessed in $90^{\circ}$ of shoulder abduction and $90^{\circ}$ of elbow flexion with the forearm in a neutral position. Shoulder girdle was manually stabilized by the primary assessor in a firm manner to avoid compensatory scapular anterior tilt (see Figure 3). HA ROM was assessed by the primary examiner passively moving each participant's shoulder into a horizontally adducted position while the glenohumeral joint was maintained in neutral rotation and scapula stabilized (see Figure 3). The intrareader reliability of these ROM measurements was reported to be good by a previous research [18].

In the first testing sessions, following baseline measurements, the two assessors left the room, and participants performed a randomly chosen stretching technique (either cross-body stretch in neutral rotation or in 90-degree ER). Excel 2016 (Microsoft, USA) was used to generate a random allocation. After each stretching protocol was completed, the two testers entered the room for post-intervention measurements. Post-intervention measurements were performed in the same manner as the baseline assessments.
For the second testing sessions, participants were again assessed for baseline data in the same manner as the first session. In this session, however, the other stretch was performed by the participants themselves. The two assessors were out of the room when the stretching interventions were being performed. The information regarding the order of stretching methods were not given to the two assessors until the completion of post-intervention assessments in second sessions, which enabled blinding for assessors and minimisation of measurement bias. In both experimental sessions, immediately after stretching interventions, participants were asked to complete two written questions with regards to stretching sensation in posterior shoulder and pain felt in the shoulder, utilising visual analogue scales (VAS) (refer to Figure 4). It was verbally explained that pain does not include stretching pain in the posterior shoulder in this context. Specific locations of shoulder pain were also asked verbally and recorded.

All testing sessions were conducted in the same laboratory room of Tokyo University of Technology at the same temperature of 26 degrees Celsius. Participants were instructed to wear the same short-sleeve T-shirts in the experiments. This maintained patient modesty for a female participant, and thus helped maximising the recruitment of participants. Care was taken to ensure that all participants received the same verbal instructions and visual cues to reduce confounding bias.

\section{Statistical analysis}

The results are shown as mean \pm standard deviation (SD). The baseline data in the two testing sessions were utilised to calculate intraclass correlation coef-
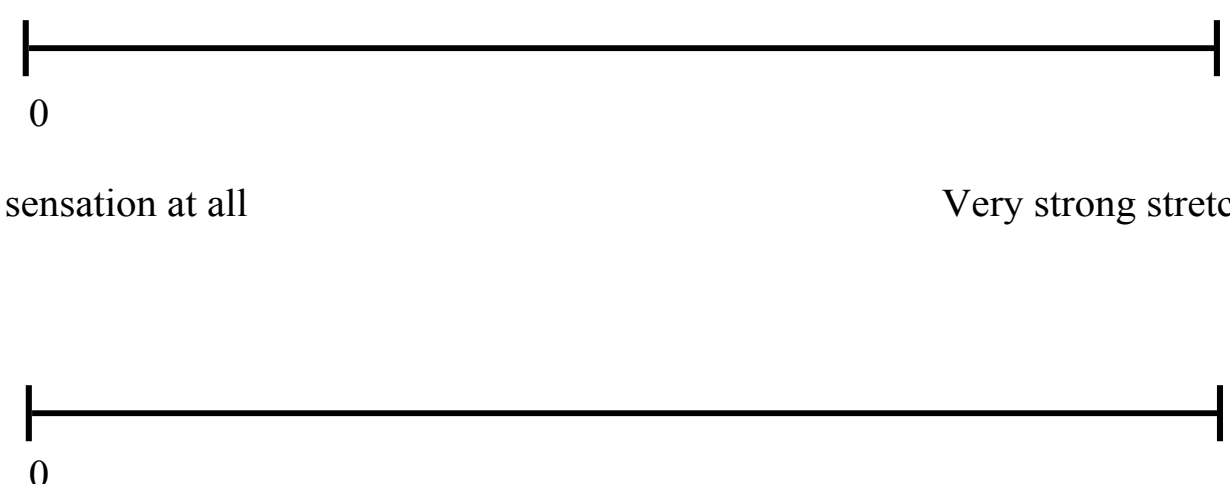

No pain at all

Worst imaginable pain

Figure 4: VAS assessment to measure stretching sensation in posterior shoulder and shoulder pain.

Table 1: Results in ROM measurements and subjective assessments for stretching sensation and pain.

\begin{tabular}{|l|l|l|l|l|l|l|}
\hline & IR pre & IR post & HA pre & HA post & Stretch & Pain \\
\hline CBS $^{\circ}$ & $43.3 \pm 9.9^{\circ}$ & $44.9 \pm 11.5^{\circ}$ & $-3.1 \pm 10.3^{\circ}$ & $-2.6 \pm 12.2^{\circ}$ & $44.8 \pm 22.6$ & $21.1 \pm 18.3^{*}$ \\
\hline${\text { CBS } 90^{\circ}}^{\circ}$ & $47.6 \pm 8.2^{\circ *}$ & $50.2 \pm 9.8^{\circ *}$ & $-4.1 \pm 11.6^{\circ}$ & $-4.3 \pm 11.8^{\circ}$ & $42.4 \pm 14.2$ & $6.9 \pm 12.5^{*}$ \\
\hline
\end{tabular}

CBS $0^{\circ}=$ cross-body stretch in neutral rotation; CBS $90^{\circ}=$ cross-body stretch in 90-degree ER pre $=$ pre-intervention data; post $=$ post-intervention data; stretch $=$ stretching sensation in posterior shoulder during stretches; ${ }^{*}=$ statistically significant improvement compared to pre-intervention data or between the two groups $(p<0.05)$. 
ficient (ICC) and determine the reliability of the ROM measurements for IR and HA. Independent t-tests were performed for two baseline data set to confirm baseline comparability. Paired t-tests were performed for differences between pre- and post-intervention data within each group [21]. Independent t-tests were used to assess differences between the two stretching conditions [21]. Statistical tests were conducted with SPSS (IBM, USA). Differences were statistically significant at $p<0.05$. Hedges' $g$ and $95 \%$ Confidence Intervals $(\mathrm{Cl})$ were calculated to determine within-group effect sizes in improving IR and HA ROM [22]. Effect sizes were categorised as large $(>0.8)$, moderate $(>0.5)$ or small $(>$ $0.2)[23]$.

\section{Results}

A mean deficit in IR ROM among 10 participants was $19.5 \pm 2.8^{\circ}$. All participants $(n=10)$ completed both testing sessions and there was no dropout (see Figure 1). ICC of the ROM measurements for IR and HA in this study were 0.89 and 0.95 respectively. Descriptive statistics for the results are summarised in Table 1 . There was no significant difference between each baseline ROM data in the two stretching groups, which confirmed the baseline comparability ( $p=0.33$ in IR, $p=0.85$ in HA). Evaluation of the within-group data using paired t-tests showed statistically significant improvements in IR ROM only after cross-body stretch in 90-degree ER $(p<0.05)$ (refer to Table 1). There was no significant difference in HA ROM between baseline and post-intervention data in the two groups.

In terms of the between-group comparisons, independent t-tests demonstrated no significant difference in changes in ROM for IR or HA between the two groups ( $p$ $=0.38$ and $p=0.68$ respectively) (refer to Table 1 ). There was no statistically significant difference in stretching sensation felt during stretches in VAS $(p=0.79)$. Participants who performed cross-body stretch in neutral rotation felt significantly more pain in VAS than those who performed cross-body stretch in 90-degree ER ( $p<$ 0.05 ) (refer to Table 1). In fact, only three out of 10 participants felt anterior shoulder pain during cross-body stretch in 90-degree ER, whereas most participants ( $n=$ 8) felt pain during cross-body stretch in neutral rotation; anterior shoulder pain $(n=6)$, lateral shoulder pain $(n=$ 1 ) and deep shoulder pain $(n=1)$.

Cross-body stretch in neutral rotation displayed small within-group effect sizes; $0.28(95 \% \mathrm{Cl}-3.70$ to 4.25) for IR ROM and 0.16 ( $95 \% \mathrm{Cl}-3.75$ to 4.44$)$ for HA ROM respectively. Cross-body stretch in 90-degree ER also showed small effect sizes; $0.28(95 \% \mathrm{Cl}-3.69$ to 4.25) for IR ROM and 0.23 ( $95 \% \mathrm{Cl}-3.34$ to 3.81$)$ for HA ROM respectively.

\section{Discussion}

The aim of this study was to compare the acute effects of two different types of cross-body stretches on
GIRD and PST and determine the influences of glenohumeral rotational angles. To the best of the author's knowledge, this is the first study investigating the influences of glenohumeral rotational angles on the outcomes of cross-body stretch. The findings of this research suggest that cross-body stretch might be more effective to improve GIRD and less likely to be provocative when it is performed in 90-degree ER compared to neutral rotation. The findings also suggest that either form of cross-body stretch may not be effective to improve PST immediately.

Generally, IR is the direction which can stretch posterior shoulder tissues [24]. Thus, it is intuitive to assume that cross-body stretch in neutral rotation can put more tensile stress to posterior shoulder tissues because it places glenohumeral joint into relatively internally rotated position compared to cross-body stretch in 90-degree ER. However, our findings in this study were against this theoretical assumption. One potential explanation may be related to increased pain due to exaggerated impingement mechanisms. It has been suggested that horizontal adduction ( $\mathrm{HA}$ ) in glenohumeral IR position can lead to increased subacromial contact pressure [25]. A combination of HA and IR position may also cause subcoracoid impingement, which is thought to be an impingement of rotator cuff (particularly subscapularis muscle), long head of biceps and joint capsule between the coracoid process and the humerus [26]. Although these impingement mechanisms may be physiological phenomenon which occurs in many asymptomatic subjects, mechanical overload can result in symptoms and it is reasonable to think that excessive compressive stress can create temporary nociceptive pain when shoulder is placed into a maximum HA in relative IR [27]. Therefore, increased subacromial and subcoracoid contact pressure might have restricted HA ROM during the stretch, leading to less stretching effects on posterior shoulder tissues in cross-body stretch in neutral rotation. This can also explain why this form of stretching produced significantly more shoulder pain compared to the other stretch (see Table 1). Since most participants $(n=6)$ felt pain in anterior shoulder, it can be speculated that cross-body stretch in neutral rotation caused excessive subcoracoid impingement and nociceptive pain, even among participants who were asymptomatic in their daily living.

Statistical comparison of within-group data showed a significant improvement only in IR ROM after cross-body stretch in 90-degree ER. This finding might be associated with sample characteristics. Although some studies found that cross-body stretch can be effective to improve GIRD and PST, the author found that the participants recruited in these studies had less limited IR and HA ROM compared to those in the present study $[18,20,28]$. Differences in sample characteristics might have confounded the results in this study. Furthermore, previous studies which reported the positive short-term effects used 
cross-body stretch with elbow extended [18,29], whereas elbow joint was flexed so that rotational angles of glenohumeral joint were monitored easily by participants in this study. This difference might have affected the length of lever in horizontal adduction (HA) moment and stretching effects. Lastly, due to the nature of the pilot study, a small sample size might have led to insufficient statistical power to detect statistical differences.

It should be noted that there are several methodological limitations and flaws in this study. A first weakness exists in the sampling method. Since participants with various sporting experiences were recruited through convenience sampling, this heterogeneity might have potentially biased our findings. A gender proportion was also skewed (nine men and one woman). Potential existence of humeral retrotorsion among participants who has played overhead sports at their younger ages might have also biased the findings because GIRD due to humeral retrotorsion will not respond to stretching interventions. Although this pilot study with a small sample size may have compromised the statistical precision to detect a potential statistical significance, further study with larger sample size will overcome this methodological limitation. Since only healthy participants without shoulder pain were recruited in this study, the findings might not be directly applicable to patients with painful shoulders. However, the findings could be applied to asymptomatic adults who need to improve GIRD and prevent shoulder injury. Although the two examiners were blinded, the absence of a genuine control group with placebo intervention or no intervention might have introduced measurement bias. Furthermore, we allowed all participants to wear their own short-sleeve T-shirts during the measurement sessions. ROM measurements for PST were potentially affected by the resistance from T-shirts being stretched. Another limitation of this study are the effect sizes of the two stretching groups. These effect sizes were consistently small to improve GIRD and PST, and $95 \% \mathrm{Cl}$ of effect sizes included the value of zero. We need to consider these potential limitations to interpret the findings of the study with caution.

\section{Conclusions}

The findings of this randomised controlled crossover pilot trial suggest that cross-body stretch might be more effective to improve GIRD and less provocative when it is performed in 90-degree glenohumeral ER compared with neutral rotation for asymptomatic adults with GIRD. The findings also suggest that either form of cross-body stretch may not be effective to improve PST immediately. Further study with a larger sample size is required to achieve stronger evidence with better statistical precision and consider its clinical implication.

\section{Acknowledgement}

The author would like to thank Tokyo University of Technology for funding this research project.

\section{Funding}

This work was financially supported by Tokyo University of Technology.

\section{Conflicts of Interest}

None declared.

\section{References}

1. Laudner KG, Moline MT, Meister K (2010) The relationship between forward scapular posture and posterior shoulder tightness among baseball players. Am J Sports Med 38: 2106-2112.

2. Giles K, Musa I (2008) A survey of glenohumeral joint rotational range and non-specific shoulder pain in elite cricketers. Phys Ther Sport 9: 109-116.

3. Challoumas D, Stavrou A, Dimitrakakis G (2017) The volleyball athlete's shoulder: biomechanical adaptations and injury associations. Sports Biomech 16: 220-237.

4. Torres RR, Gomes JL (2009) Measurement of glenohumeral internal rotation in asymptomatic tennis players and swimmers. Am J Sports Med 37: 1017-1023.

5. Shanley E, Thigpen CA, Clark J, Wyland DJ, Hawkins RJ, et al. (2012) Changes in passive range of motion and development of glenohumeral internal rotation deficit (GIRD) in the professional pitching shoulder between spring training in two consecutive years. J Shoulder Elbow Surg 21: 16051612.

6. Myers JB, Oyama S, Wassinger CA, Ricci RD, Abt JP, et al. (2007) Reliability, precision, accuracy, and validity of posterior shoulder tightness assessment in overhead athletes. Am J Sports Med 35: 1922-1930.

7. Harshbarger ND, Eppelheimer BL, McLeod TCV, McCarty CW (2013) The effectiveness of shoulder stretching and joint mobilizations on posterior shoulder tightness. J Sport Rehabil 22: 313-319.

8. Kibler WB, Sciascia A, Thomas SJ (2012) Glenohumeral internal rotation deficit: pathogenesis and response to acute throwing. Sports Med Arthrosc Rev 20: 34-38.

9. Gates JJ, Gupta A, McGarry MH, Tibone JE, Lee TQ (2012) The effect of glenohumeral internal rotation deficit due to posterior capsular contracture on passive glenohumeral joint motion. Am J Sports Med 40: 2794-2800.

10. Hung CJ, Hsieh CL, Yang PL, Lin JJ (2010) Relationship between posterior shoulder muscle stiffness and rotation in patients with stiff shoulder. J Rehabil Med 42: 216-220.

11. Chant CB, Litchfield R, Griffin S, Thain LM (2007) Humeral head retroversion in competitive baseball players and its relationship to glenohumeral rotation range of motion. J Orthop Sports Phys Ther 37: 514-520.

12. Tyler TF, Nicholas SJ, Roy T, Gleim GW (2000) Quantification of posterior capsule tightness and motion loss in patients with shoulder impingement. Am J Sports Med 28: 668-673.

13. Muraki T, Yamamoto N, Zhao KD, Sperling JW, Steinmann SP, et al. (2012) Effects of posterior capsule tightness on subacromial contact behavior during shoulder motions. J Shoulder Elbow Surg 21: 1160-1167.

14. Werner SL, Gill TJ, Murray TA, Cook TD, Hawkins RJ (2001) Relationships between throwing mechanics and shoulder distraction in professional baseball pitchers. Am J Sports Med 29: 354-358. 
15. Shanley E, Rauh MJ, Michener LA, Ellenbecker TS, Garrison JC, et al. (2011) Shoulder range of motion measures as risk factors for shoulder and elbow injuries in high school softball and baseball players. Am J Sports Med 39: 19972006.

16. Tyler TF, Nicholas SJ, Lee SJ, Mullaney M, McHugh MP (2010) Correction of posterior shoulder tightness is associated with symptom resolution in patients with internal impingement. Am J Sports Med 38: 114-119.

17. Mine K, Nakayama T, Milanese S, Grimmer K (2017) Effectiveness of Stretching on Posterior Shoulder Tightness and Glenohumeral Internal-Rotation Deficit: A Systematic Review of Randomized Controlled Trials. J Sport Rehabil 26: 294-305.

18. Mine K (2017) Immediate effects of two types of stretching techniques on glenohumeral internal rotation deficit and posterior shoulder tightness; a crossover randomised controlled trial. Journal of Physical Therapy and Sports Medicine 1.

19. Wilk KE, Hooks TR, Macrina LC (2013) The modified sleeper stretch, and modified cross-body stretch to increase shoulder internal rotation range of motion in the overhead throwing athlete. J Orthop Sports Phys Ther 43: 891-894.

20. McClure P, Balaicuis J, Heiland D, Broersma ME, Thorndike CK, et al. (2007) A randomized controlled comparison of stretching procedures for posterior shoulder tightness. J Orthop Sports Phys Ther 37: 108-114.

21. Norman G (2010) Likert scales, levels of measurement and the "laws" of statistics. Adv Health Sci Educ Theory Pract 15: $625-632$
22. Hedges LV (1982) Estimation of effect size from a series of independent experiments. Psychological Bulletin 92: 490499.

23. Kazis LE, Anderson JJ, Meenan RF (1989) Effect sizes for interpreting changes in health status. Med Care 27: S178-S189.

24. Izumi T, Aoki M, Muraki T, Hidaka E, Miyamoto S (2008) Stretching positions for the posterior capsule of the glenohumeral joint: strain measurement using cadaver specimens. Am J Sports Med 36: 2014-2022.

25. Tucker S, Taylor NF, Green RA (2011) Anatomical validity of the Hawkins-Kennedy test--a pilot study. Man Ther 16: 399-402.

26. Okoro T, Reddy V, Pimpelnarkar A (2009) Coracoid impingement syndrome: a literature review. Curr Rev Musculoskeletal Med 2: 51-55.

27. Lewis JS (2010) Rotator cuff tendinopathy: a model for the continuum of pathology and related management. $\mathrm{Br} \mathrm{J}$ Sports Med 44: 918-923.

28. Manske RC, Meschke M, Porter A, Smith B, Reiman M (2010) A randomized controlled single-blinded comparison of stretching versus stretching and joint mobilization for posterior shoulder tightness measured by internal rotation motion loss. Sports Health 2: 94-100.

29. Hammons EdD D, Curtin M, Pfeiffer ED (2015) A Randomized and Blinded Study for the Treatment of Glenohumeral Internal Rotation Range of Motion Restriction: The Prone-Passive Stretching Technique. Journal of Sports Medicine and Allied Health Sciences: Official Journal of the Ohio Athletic Trainers Association 1: 5. 